\title{
Concepto y aplicación práctica que dan los profesores de química a la evaluación final, análisis de falencias y potencialidades que tiene en el proceso de enseñanza y de aprendizaje
}

Concept and practical application of chemistry teachers give to the final evaluation,
analysis and potential flaws in the process of teaching and learning

Martínez Diana E. y Giraldo Liselly

Profesoras en formación inicial. Universidad Pedagógica Nacional, Calle 73 No 11-95 Bogotá D.C. Colombia. Facultad de Ciencia y Tecnología. Departamento de Química.

dayanam 332@hotmail.com, lisgirsal 14@hotmail.com

\section{Resumen}

Con el objetivo de analizar qué imagen, concepto y aplicación práctica respecto a la evaluación final tienen los profesores de nivel universitario, se realiza una pequeña indagación en la UPN, con docentes del departamento de química. Se busca entonces evidenciar algunas de las falencias y potencialidades que tiene la evaluación final en el proceso de enseñanza y de aprendizaje, con relación a tal visión, cuando finalmente esta, es el resultado de la planeación previa del profesor.

Se crean una serie de instrumentos y espacios de trabajo, ayo objeto es poner de manifiesto tales concepciones, estos hacen parte del diseño de una serie de etapas a través de las cuales se espera llegar producto de la indagación y de la consulta bibliográfica, a proponer una serie de características que debe poseer la evaluación final, como herramienta eficaz del profesor, para evidenciar el desempeño y progreso de los estudiantes en un momento determinado.

Palabras claves

Evaluación final, concepciones, registro de evaluación continua, diseño de actividades especiales, proyecto, examen, rol del profesor, dimensiones de la enseñanza, competencias de los profesores.

Abstract

In order to analyze this image, concept and practical application regarding the final assessment teachers have university-level inquiry is conducted in a small UPN, with teachers of the department of chemistry. It then seeks to highlight some of the shortcomings and potential of the final evaluation in the process of teaching and leaming in relation to that vision, when finally this is the result of planning on the teacher. This creates a series of tools and work spaces, which aims to highlight these concepts, these are part of the design of a series of stages through which the product is expected to arrive in the inquiry and of literature, to propose A number of features that should have the final evaluation of theteacher as an effective tool to demonstrate the performance and progress of students at any given time.

\section{Keywords}

Final evaluation, concepts, recording of continuous assessment, design of special events, project, review, role of the teacher, the dimensions of teaching competence of teachers. 


\section{Marco teórico}

La evaluación final ofrece un informe detallado que da cuenta del rendimiento de los estudiantes en un momento dado, generalmente al final de un período académico, representa un indudable progreso si se le comprende también como instrumento de la mejora de la enseñanza, es decir, si se pretende hacer de ésta un instrumento de seguimiento y mejora del proceso, por ello es necesario situarla como una actividad colectiva en la que el papel tanto del profesor como del centro, son factores determinantes (Gil,1993).

Por su naturaleza la evaluación final debe proporcionar información a los profesores, padres de familia, estudiantes y a la institución misma acerca del desempeño y progreso de los educandos, por ende no debe ser una prueba de la eficacia del sistema educativo, sino que, se debe constituir una forma de proporcionar información eficaz para el diseño de plan de estudios (Bruner, 1966). De esta forma se constituye en la base de la toma decisiones, referente a la certificación; de ahí deriva su carácter sumativo.

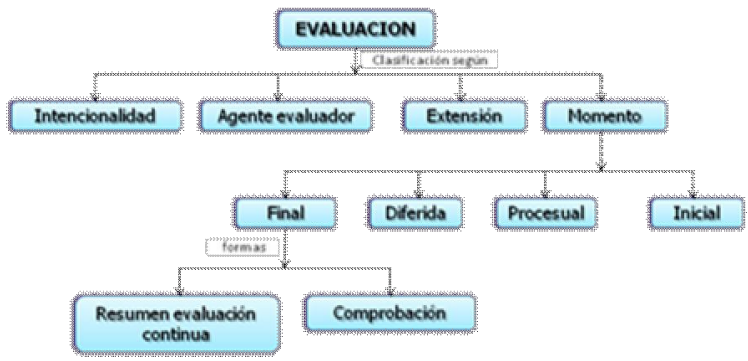

Como señala (Harlen, 1989) en su libro Enseñanza y Aprendizaje de las ciencias existen dos formas de concebir la evaluación final:

$>$ Resumen de los registros de la evaluación continua.

> Comprobación a través de tareas especiales orientadas a revelar el grado de desarrollo de algunas destrezas 0 conocimientos.

El resumen

Permite:

$>$ Tener en cuenta la evaluación continua

$>$ Realizar observaciones finales sobre el avance entomo al compendio de conocimientos, destrezas y actitudes trabajadas.

$>$ Aspectos menos favorables del resumen:

$>$ Si las temáticas tratadas al principio no han sido objeto de repaso parte de la información puede quedar en el olvido.

$>$ Dificultad de elaborar un resumen descriptivo que permita comparar el rendimiento de los estudiantes (diferencias en las oportunidades de aprendizaje).

$>$ El registro intenta recoger la variedad de realizaciones de los estudiantes mediante la descripción y el archivo de las muestras de trabajo.

Comprobación

$>$ Otorga una oportunidad igualitaria para mostrar lo que se esta en capacidad de hacer con relación a un conjunto de experiencias.

> Proporcionar tareas iguales no es lo mismo que darles las mismas oportunidades, la oportunidad de aprendizaje consiste en el ajuste entre la actividad y la capacidad del estudiante. (Harlen, 1989)

\section{Desventajas:}

$>$ Limitación de las tareas al contexto del test, sólo se induyen un conjunto restringido de destrezas y conocimientos. 
> Algunas destrezas sólo se muestran en actividades que se extienden en el tiempo.

$>$ Resta tiempo al aprendizaje.

$>$ Induce ansiedad.

La evaluación final tiene diferentes maneras de concebirla y aplicarla, es decir que existen diversas Reflexiones sobre la evaluación que permiten replantear su función y formas habituales, además, integrarla de forma eficiente en los nuevos modelos de enseñanza aprendizaje de las ciencias, como un proceso de verdadera investigación (Bruner, 1966), un ejemplo daro e innovador de ello son las discusiones, debates, plenarias entre otros, en donde existe el planteamiento de una situación problema, lo que se convierte en algo más que calificaciones de tipo tradicional, ya que trata de analizar la situación problema, los puntos de vista, la reconstrucción de conceptos y la generación de nuevos interrogantes.

Al existir interacción entre docente-estudiante $y$ estudiante-estudiante, el profesor puede observar como piensa el estudiante, con que ideas e instrumentos representa el fenómeno, como lo aborda e indaga hasta llegar a su comprensión (Flores, 1999).

\section{Metodología}

La unidad de observación seleccionada son cinco profesores del departamento de química de la Universidad Pedagógica Nacional y tres cursos de estudiantes (Teońas Químicas III, Sistemas Inorgánicos I, Sistemas Orgánicos II, segundo semestre 2008 total estudiantes: 51). Se hará uso de la población estudiantil como parte integral de nuestra indagación, ya que puede aportar herramientas oportunas para el mejoramiento del proceso educativo en el marco de las evaluaciones finales; a través de la consulta de la opinión de los estudiantes, el profesor podrá conocer cómo entienden y que esperan de la evaluación final.

La muestra poblacional que será objeto de nuestro estudio consistirá en dos grandes grupos: profesores de química y estudiantes que reciben de ellos dases. No se harán diferencias entre las distintas materias de la disciplina (por ejemplo: sistemas orgánicos o inorgánicos, teorías químicas, etc.) se recogerán las opiniones en general de los estudiantes.

\section{Situación problema}

¿Cuál es el concepto y forma de aplicar la evaluación final de los profesores de química y cómo incide en el desarrollo del proceso de enseñanza y de aprendizaje (falencias y potencialidades)?

\section{¿Qué se hizo?}

La indagación va a ser dividida en dos grandes etapas:

Primera etapa o de pre-test

Primera parte

> Diseño de los dos auestionarios (Tanto para profesores como para estudiantes).

$>$ Mediante el diagnóstico (pre-test) se busca identificar en los profesores las ideas previas con respecto a la evaluación final.

$>$ Aplicación de instrumentos que nos permitan recoger datos con el objetivo de sustentar las hipótesis planteadas para el desarrollo de la indagación.

$>$ Análisis de instrumentos y sistematización de los mismos.

\section{Segunda parte}

$>$ Realización de post-test.

$>$ Presentación de la información (pequeña exposición del tema de los profesores) lo que puede o no cambiar su punto de vista respecto a las evaluaciones finales. (anexo 5)

\section{Segunda etapa}


> Emisión de juicios de valor orientados a proponer la optimización y mejoramiento de los resultados de las evaluaciones finales en el proceso de enseñanza y de aprendizaje.

Hipótesis dirigidas a los profesores

$>$ Los profesores de la UPN del departamento de química usan el examen como una actividad para realizar la evaluación final.

$>$ Los profesores de la UPN del departamento de química con fines de realizar la evaluación final elaboran un resumen de los registros de la evaluación continua.

$>$ Los profesores de la UPN del departamento de química realizan la evaluación final como proyecto, de manera que se convierte en un elemento de trabajo habitual.

$>$ Los profesores de la UPN del departamento de química prefieren para la evaluación final la asignación de un trabajo correspondiente al ultimo núcleo temático del curso

$>$ Los profesores de la UPN del departamento de química optan por actividades altemativas como: plenarias, debates etc, para ejecutar la evaluación final

Hipótesis dirigida a estudiantes

> El estudiante tiene actitud positiva frente a la evaluación final porque considera que a través de ella puede dar cuenta de la evolución de su proceso de aprendizaje, de lo que realmente sabe.

> El estudiante tiene una actitud desfavorable frente a la evaluación final porque considera que no es conveniente para el registro de sus notas.

$>$ Cuestionarios (Anexo 1) (Anexo 2) (basados en Gallego y Pérez ,2002)

$>$ Los cuestionarios están formados de dos partes: Una primera parte consiste en el diseño de una escala de actitudes tipo Likert en la cual por medio de afirmaciones queremos saber la actitud tanto favorable y desfavorable respecto de las evaluaciones finales.

La segunda parte esta conformada por preguntas abiertas, donde se pretende indagar algo más acerca del punto de vista tanto de profesores como estudiantes acerca de a las evaluaciones finales.

\section{Resultados}

Pruebas profesores (anexo 3)

Primera parte

Con el objetivo de caracterizar la evaluación final concebida y aplicada por los profesores de química del departamento, se diseño una prueba tipo Likert a través de la cual se pretenden determinar tendencias respecto a las actividades propuestas para la evaluación, se sistematizaron y promediaron las puntuaciones para las afirmaciones propuestas de acuerdo a este esquema:

> Examen que mide la reproducción de conceptos y ejercicios, (asimilación memorística): preguntas $1,5,9$

> Proyecto final donde el estudiante puede poner de manifiesto el desarrollo de los conceptos, sus interrelaciones y el manejo interdisciplinar de los mismos: preguntas 3,7,11

$>$ Plenaria o debate en el cual el estudiante puede expresar su posición frente a lo aprendido: preguntas 4, 8, 12

$>$ Resumen del registro de la evaluación continua: 2, 6,10.

\begin{tabular}{|c|c|c|c|c|}
\hline Actividad & Examen & P. final & Exposición & Registro \\
\hline Valor promedio & 2.8 & $\mathbf{4 . 4 2}$ & $\mathbf{4 . 0 4}$ & $\mathbf{2 . 2 2}$ \\
\hline
\end{tabular}

Tabla No. 1 resultados promedios globales obtenidos en las pruebas tipo Likert para profesores.

Segunda parte 
Como complemento se planteo una serie de preguntas abiertas, de las que se pudo extraer con base en el diseño de una matriz de valoración los siguientes resultados:

1. Actividades que se proponen para la evaluación final y porcentaje de utilización:

$>$ Pruebas escritas tradicionales, nombrada el $50 \%$ de las veces.

$>$ Promedio de las evaluaciones parciales, nombrada el $0 \%$ de las veces

$>$ Presentaciones orales (plenarias, debates, seminarios, discusiones, entre otros.), nombrada el $1.25 \%$ de las veces.

$>$ Proyectos que reúnan el dominio de todos los contenidos del aprendizaje, nombrada el $37.5 \%$ de las veces.

2. Características que debe poseer la evaluación final:

$>$ Conceptualmente concreta

> Permitir evidenciar un lenguaje propio.

$>$ De considerar cambio de conducta (actitudinal)

> Presentar el desarrollo de competencias, destrezas y habilidades.

$>$ Interdisciplinar.

3. Medidas que se pueden tomar respecto a los resultados de la evaluación final:

$>$ Retroalimentación.

$>$ Mejorar la metodología

> Buscar propuestas altemativas mediante la implementación de estrategias didácticas

$>$ Implementar el auto el $\infty$ y heteroevaluación.

$>$ Mostrar un informe acerca del desempeño.

$>$ Realizar otro tipo de evaluación acorde con los temas y necesidades de los estudiantes.

Pruebas estudiantes (anexo 4)

Con el objetivo complementar esta indagación se consulto la opinión de los estudiantes con el propósito de establecer características de su forma de concebir y su actitud frente a la evaluación final.

\section{Primera parte}

Corresponde al test tipo Likert en el que se pretende conocer la posición actitudinal del estudiante.

Se sistematizaron y promediaron las puntuaciones para las afirmaciones propuestas de acuerdo a este esquema:

$>$ Posición favorable: preguntas 1, 3, 5,7.

$>$ Posición desfavorable: 2, 4, 6,8.

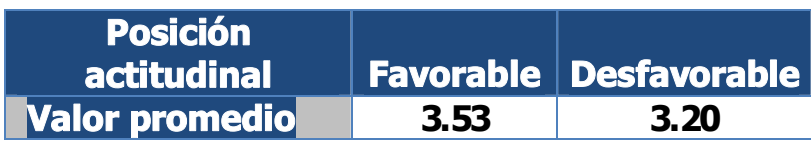

Tabla No. 2 resultados promedios globales obtenidos en las pruebas tipo Likert para estudiantes.

\section{Segunda parte}

Como complemento se planteo una serie de preguntas abiertas, de las que se pudo inferir con base en el diseño de una matriz de valoración los siguientes resultados:

1. Cómo se concibe la evaluación final:

$>$ Evaluación que reúne, recopila los contenidos y todos los conocimientos adquiridos durante un periodo de tiempo, nombrada el $50.98 \%$ de las veces

$>$ Determinación de logros alcanzados, nombrada el $1.96 \%$ de las veces

> Henramienta que involucra aspectos generales y específicos elaborados en el proceso académico, nombrada el $3.92 \%$ de las veces 
Actividades espećficas en las que se busca observar el nivel de destrezas adquiridas, nombrada el $5.88 \%$ de las veces

$>$ Revisión conceptual, nombrada el 3.92\% de las veces

> Instrumento para mirar el aprendizaje significativo, induyendo todas las temáticas vistas en el programa académico, nombrada el $13.72 \%$ de las veces.

$>$ Proceso que permite evidenciar falencias tanto de estudiantes como de docentes con miras a mejorar el proceso de educación, nombrada el $11.76 \%$ de las veces.

$>$ Conjunto de conocimientos adquiridos y aplicados en problemas cotidianos, nombrada el 1.96\% de las veces.

$>$ Examen (oral, escrito, entre otros) que permite corroborar la asimilación de conocimientos, nombrada el $5.88 \%$ de las veces

2. Tipos de actividades cómo se aplica frecuentemente la evaluación final:

$>$ Evaluadiones escritas (parciales). Nombrada el $56.41 \%$ de las veces.

$>$ Proyecto. Nombrada el $\mathbf{2 1 . 7 9 \%}$ de las veces.

$>$ Talleres y cuestionarios. Nombrada el $11.54 \%$ de las veces.

$>$ Sustentaciones (exposición). Nombrada el $\mathbf{8 . 9 7 \%}$ de las veces

$>$ Resolución de problemas. Nombrada el $1.28 \%$ de las veces

3. dComo sienten los estudiantes respecto a estas actividades?:

$>$ Es considerada favorable. 29 personas

$>$ Es considerada desfavorable. 21 personas

$>$ Se muestra indiferente. 1 persona.

\section{Análisis de resultados}

\section{Pruebas profesores}

\section{Primera parte}

La tabla No 1 muestra los resultados generales que se obtuvieron en el análisis de las pruebas tipo Likert aplicadas a profesores. Se analizaron las tendencias de acuerdo a la lógica de los promedios para estas pruebas.

Deducciones que se pueden realizar acorde con las puntuaciones:

De acuerdo con la dasificación previamente acordada en la cual se hace diferencia entre evaluación final como resumen del registro de la evaluación continua y como corroboración a través de tareas especiales, se puede observar que respecto a las primeras existe una tendencia al desacuerdo, puesto que sus puntuaciones se ubican entre 2 y 2.5. Por otra parte en lo referente a las actividades de comprobación se puede apreciar que son los proyectos las actividades que prefieren los profesores ya que las puntuaciones se encuentran entre 4 y 5 . También se observó una dara tendencia a la realización de exposiciones lo que se muestra con puntuaciones entre 4 y 5, por el contrario se muestra poco acuerdo con las características del examen final, puntuaciones entre 2 y 3.

\section{Segunda parte}

Respecto a las actividades de comprobación se muestra una clara preferencia de los docentes a aplicar con sus estudiantes pruebas escritas de tipo tradicional.

Encontramos que dentro de las ventajas que ofrece se encuentran:

$>$ Igualdad de oportunidades.

> El docente puede constatar que es el estudiante quien la realiza.

> Aproxima al estudiante al repaso delas temáticas. 
Sin embargo pueden surgir desventajas en cuanto a:

$>$ Se estimula la superficialidad si no se da interiorización de los conocimientos.

$>\quad$ No permiten saber cuanto se ha aprendido.

$>$ Solo se pueden demostrar ciertas destrezas.

En segundo lugar de preferencia se encuentran los proyectos, por tanto es importante resaltar que a través de estos, el estudiante puede encontrar un contexto aplicativo de todos los conocimientos adquiridos. A partir de ellos se desarrollan competencias tanto básicas, como cientúficas; además estimulan el trabajo tanto individual como colectivo.

Por su parte las presentaciones orales en las que se encuentran menores tendencias, tienen como ventaja ser un complemento del trabajo escrito (Cárdenas, Ladino \& Zapata, 2003) y promover el manejo del público, preparando al estudiante para desarrollar respuestas consecuentes con los temas.

En cuanto a la determinación de las características que debe poseer la evaluación final los profesores proponen una serie de aspectos que consideramos de gran relevancia, a estas condiciones se quieren anexar las siguientes:

$>$ Contener resolución de problemas prácticos que se puedan desarrollar en contextos simultáneos.

$>$ Considerar todos los contenidos: contenido conceptual (saber), procedimental (saber hacer) y actitudinal (saber ser)

$>$ Favorecer el desarrollo de competencias, destrezas y habilidades

$>$ Ser interdisciplinaria.

Por último, al establecer medidas que se pueden tomar respecto a los resultados de la evaluación final, se suman junto a las que han sido planteadas por los profesores, algunas otras con el objetivo de complementar esta reflexión. Entre estas:

$>$ Flexibilidad y manejo del tiempo.

> Establecimiento de las condiciones de la evaluación.

> Formular preguntas en las que la memoria sea parcialmente necesaria. (Cárdenas, Ladino \& Zapata, 2003).

\section{Conclusiones}

$>\quad$ Las pruebas realizadas nos permiten responder la pregunta eje de nuestro proyecto en aranto a la forma de llevarse a cabo la evaluación final, en estas se puede observar que son los exámenes finales y los proyectos las actividades preferidas por los profesores, lo que se puede corroborar con los resultados puntuados por los estudiantes.

$>$ Respecto a las concepciones que tienen los profesores de la evaluación final se encuentra que existe una gran aproximación al referente teórico, sin embargo es necesaria la implementación de cambios que propendan a mejoras respecto al componente actitudinal, donde todavía se encuentran problemáticas que marcan grandes brechas entre lo que espera el estudiante de la evaluación y su aplicación real.

$>$ Aunque el examen escrito tenga las desventajas anteriormente mencionadas, es una de las formas de realizar la evaluación final mas ampliamente utilizada por los docentes, además de seguir siendo consideradas como fundamentales y necesarias. Sin embargo para su correcto uso se recomienda:

$>$ La revisión global que induya actividades coherentes con un aprendizaje por construcción.

> La discusión de las posibles respuestas para evidenciar la existencia de pre concepciones equivocadas. 
$>$ Que las condiciones de su realización deben ser compatibles con lo que supone una verdadera construcción de conocimiento, en particular se debe evitar que los estudiantes se vean obligados por las limitaciones del tiempo (Rasco, 1995).

A continuación señalamos las potencialidades que consideramos tiene la evaluación final tanto para la enseñanza como para el aprendizaje

$>$ Utilizarse como herramienta para regular la calidad del proceso de enseñanza, mejorar sobre lo que se esta trabajando o plantear nuevas estrategias de evaluación final en el proceso de enseñanza y de aprendizaje.

$>$ Permitir observar como todos los conceptos vistos durante el desarrollo del periodo académico son útiles.

$>$ Estar alejada del contexto al no presentar secuencia y coherencia con los temas que se han visto durante el curso.

$>$ Permitir la evaluación formativa y formadora.

Algunas características que debe tener una buena actividad de evaluación final como método comprobación

> Actividades bien diseñadas presentadas de forma atractiva y estimulantes para los niños.

$>$ Asegurarse de que aplican sus ideas las elaboran partiendo de su propio pensamiento (comprobar las ideas en evolución de los niños).

$>$ Evitar las preguntas que lleven a recordar datos

$>$ Participación de los alumnos en el establecimiento de los objetivos del aprendizaje.

$>$ El contenido debe dar la oportunidad de utilizar las destrezas en contextos diferentes

\section{Bibliografía}

Bruner, J (1966).Hacia una teońa de la instrucción. Unión tipográfica editorial hispanoamericana. Primera edición.

Cárdenas S. F. A., Ladino O. Y. y Zapata C. P. N. (2003) Reflexiones acerca de la Evaluación. En: Educación y Formación del Pensamiento Científico. Cátedra ICFES "Agustín Nieto Caballero". (pp. 169-189). Bogotá: Arfo Editores..

Flores, R (1999). Evaluación pedagógica y cognición. Capitulo VII la evaluación del aprendizaje en las áreas de ciencias y de matemáticas. (Pp. 115-137). Bogotá: Mc Graw Hill.

Gallego, R. Pérez, R (2002). El Problema del cambio en las concepciones de estudiantes de formación avanzada. Enser̃anza de las ciencias, 20 (3) pp. 401-414.

Gil, D (1993). La necesidad de innovaciones en la evaluación. En OEI (Ed.), Enseñanza de las Ciencias y la Matemática Tendencias e innovaciones (pp. 47-54). Editorial popular

Harlen, W (1989). Capitulo VIII: la evaluación: objetivos, principios y enfoques y capitulo IX técnicas de evaluación continua y final. Enseñanza y Aprendizaje de las Ciencias. (pp. 160-172), (pp. 173-198). Cuarta edición. Colombia: Ediciones Morata.

Rasco, A, (1995) "La evaluación del sistema educativo: Algunas respuestas cíticas al porqué y al cómo" en: AAW, "Volver a pensar la educación, vol. II de Prácticas y discursos educativos", Madrid: Paideia/Morata. 